\title{
Política Monetária em Tempos de Crise
}

\author{
Vladimir K. Teles*, Diogo de Prince Mendonça ${ }^{\dagger}$
}

\begin{abstract}
Contents: 1. Introdução; 2. Crise de 2008 no Brasil: Fatos Estilizados; 3. Modelo; 4. Estimação; 5. Propriedades do Modelo; 6. Aplicações; 7. Implicações para Política Monetária; 8. Conclusões; A. Apêndice A - Modelo Completo Log-Linearizado; B. Apêndice B - Posteriors Estimados.

Keywords: Sudden-Stops, Política Monetária, Termos de Troca, Fricções Financeiras.

JEL Code: E32, F32, F41.
\end{abstract}

O presente artigo busca investigar como crises causadas por choques externos, como sudden-stops e choques nos termos de troca, afetaram a economia brasileira. Para tanto propomos um modelo teórico simples de cash-in-advance com fricções financeiras de forma que choques de liquidez causam mudanças na alocação dos fatores de produção e na produtividade da economia. Em seguida, o modelo é estimado para a economia brasileira e as implicações para política monetária são analisadas.

This paper investigates how external shocks (such as sudden-stops and shocks in terms of trade) affected the Brazilian economy. Therefore we propose a simple theoretical model of cash-in-advance with financial frictions. The model is estimated for the Brazilian economy and the implications for monetary policy are analyzed.

\section{INTRODUÇÃO}

Crises financeiras, como a ocorrida em 2008, são acompanhadas por fortes quedas da produtividade total dos fatores (PTF) nos países emergentes. Tal efeito é amplamente documentado para todas as economias emergentes por Calvo et alii (2006) e Cerra (2008), para as crises nas décadas de 80 e 90 , e por Queralto (2012) para a crise de 2008. Neste artigo, mostramos evidências de que a crise de 2008 levou a uma queda da PTF no Brasil e apresentamos um modelo onde os efeitos reais da crise resultam de choques externos que implicam em mudanças nos preços relativos dos fatores de produção, e assim na PTF. Tal modelo é estimado para a economia brasileira, e as implicações para a condução de política monetária são discutidas.

Nós analisamos dois tipos de choques externos sob os quais a economia brasileira se deparou recentemente, e suas conseqüências sobre a economia: choques de sudden-stops e choques nos termos de troca. Dessa forma, o foco do artigo será avaliar a condução de políticas monetárias em períodos de crises provocadas por estes dois tipos de choques.

\footnotetext{
*Fundação Getulio Vargas (EESP/FGV-SP). E-mail: vladimir.teles@fgv . br

${ }^{\dagger}$ Fundação Getulio Vargas (EESP/FGV-SP). E-mail: dioted@gmail. com
} 
Em uma economia com friç̧ões no sistema financeiro é de se esperar certo grau de ineficiência alocativa. Uma queda de produtividade acentuada durante uma crise financeira revela que a crise pode causar um aumento desta ineficiência. Assim exploramos como um sudden-stop implica em um aumento da ineficiência alocativa causada por friç̧ões financeiras. Ao mesmo tempo, quando uma economia precisa importar insumos, um choque nos termos de troca resulta em uma mudança dos preços relativos da economia, implicando em outra fonte de ineficiência alocativa.

O modelo é desenvolvido a partir de uma mescla de insights fornecidos por modelos de working capital com cash-in-advance (e.g. Carlstrom e Fuerst, 1995), de friç̧ões financeiras em economias abertas e pequenas (e.g. Neumeyer e Perri, 2005, Pratap e Urrutia, 2012), e modelos com importação de insumos, de forma que os termos de troca são relevantes na alocação de recursos produtivos (e.g. Mendoza, 1995, Acemoglu e Ventura, 2002).

Dessa forma, o modelo consiste em uma economia em que o lado real segue um modelo padrão de Real Business Cycles (RBC). A moeda é inserida no modelo a partir de uma restrição cash-in-advance na decisão de consumo das famílias, e em uma restrição de working capital na decisão de contratação de trabalhadores pelas firmas. Tais restrições implicam em duas ineficiências sobre a economia:

1) a restrição de moeda pelas firmas faz com que os juros cobrados pelo intermediário financeiro afete o custo do trabalho, e, assim, a sua demanda (Fuerst, 1994);

2) a restrição de moeda das famílias implica em uma distorção na decisão de consumo (Cooley e Hansen, 1989).

Nós adicionamos ao modelo o setor externo de duas formas. Primeiramente, como um agente que empresta recursos para o intermediário financeiro, sendo uma fonte de liquidez, o que reduz a primeira fonte de distorção listada no parágrafo anterior; e em segundo lugar, como produzindo bens intermediários que são importados pelas firmas para serem utilizados na produção. Como resultado, os termos de troca afetam os preços relativos dos fatores, e assim, a eficiência alocativa da economia, de forma que choques nos termos de troca terão um efeito similar a um choque de produtividade.

O modelo é estimado para a economia brasileira para o período 2000-2012. A partir dos parâmetros estimados e calibrados, o modelo foi capaz de construir uma série de hiato da PTF que segue as mesmas direções da série observada nos dados, explicando boa parte do seu comportamento no período. Além disso, os resultados indicam que a regra de política monetária que maximiza o bem estar depende da fonte dos choques, uma vez que cada choque implica em distorções diferentes sobre o modelo.

\section{CRISE DE 2008 NO BRASIL: FATOS ESTILIZADOS}

A economia brasileira apresentou mudança na sua trajetória após a crise financeira de 2008. Nesta seção, a mudança na trajetória é documentada. A Figura 1 apresenta a série de PIB (Produto Interno Bruto) do Brasil trimestralmente entre 2006 e 2012. O produto brasileiro apresentava uma trajetória de crescimento até o final de 2008, quando a crise financeira levou à diminuição do PIB. Depois o PIB retoma a trajetória de crescimento mas, a partir da segunda metade de 2010 assume uma trajetória de estagnação. Tal comportamento é bem evidente ao observarmos as séries da indústria na Figura 2 .

O ponto que chama mais a atenção nestas figuras é que fica bem visível que a produção sofreu uma queda repentina com a crise de 2008, e, longe de retornar à mesma trajetória de crescimento, começou a divergir da trajetória anterior, mesmo após quatro anos pós-crise. Tal comportamento fornece evidências de que a crise foi capaz de alterar a trajetória de crescimento do país. ${ }^{1}$

Como verificamos a crise teve como resultado uma mudança significativa e persistente na produção industrial brasileira. Na literatura de crescimento há explicações que tentam vincular a mudança de

\footnotetext{
${ }^{1}$ Para confirmar se esta mudança foi significativa basta fazer um VAR com uma dummy de pulso para o terceiro trimestre de 2008 e as taxas de crescimento. Os resultados estão apresentados em uma versão anterior publicada como working paper.
} 
Figure 1: Trajetória do PIB do Brasil

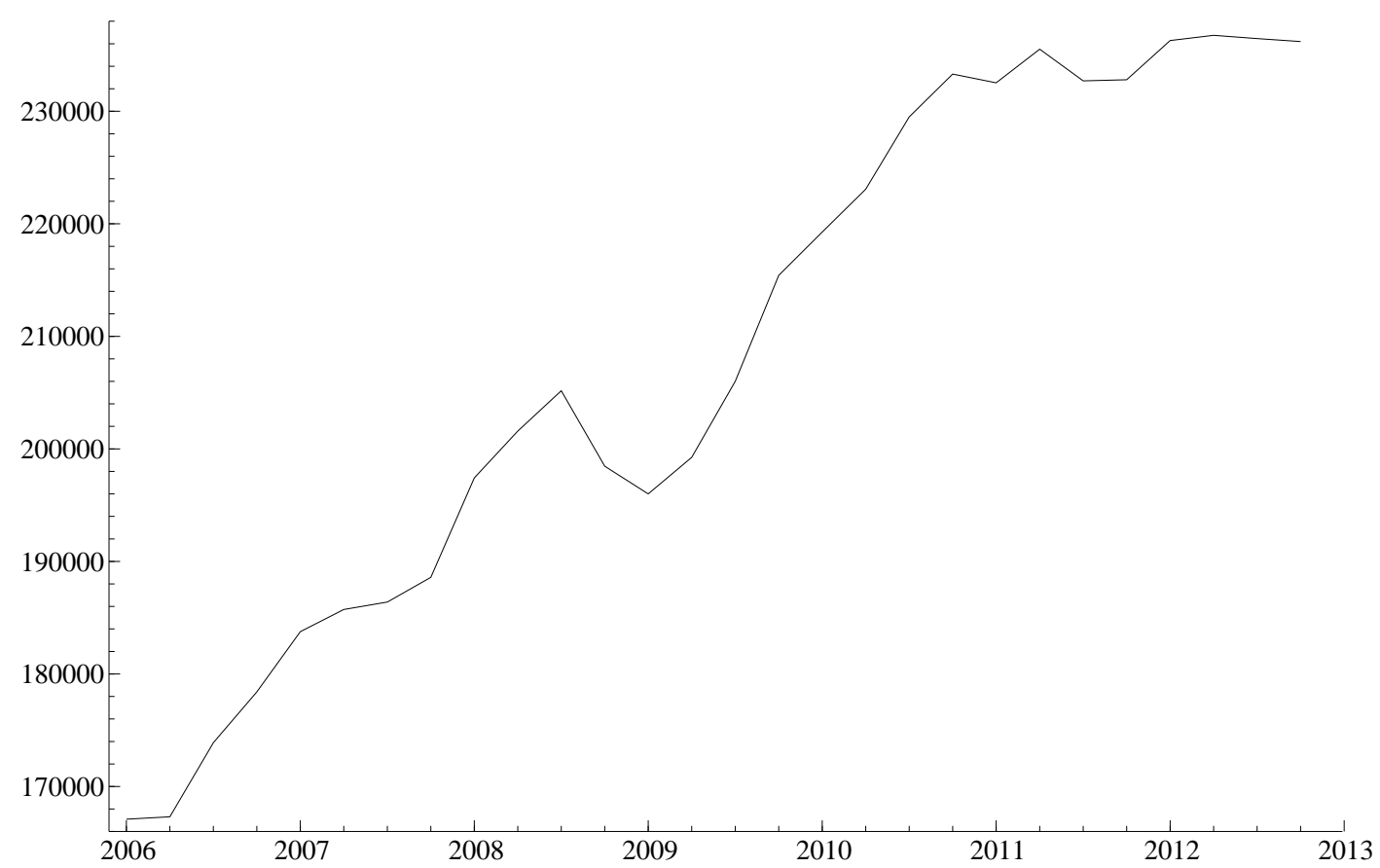

trajetórias de longo prazo em face à crises por friç̧ões financeiras em países em desenvolvimento, como em Queralto (2012), onde se incorpora o ambiente de fricções financeiras à la Bernanke-Gertler em um modelo de crescimento endógeno. No presente artigo iremos nos focar às consequências de curto prazo de crises como choques de liquidez.

Porém, outro aspecto da crise chama a atenção. O comportamento de queda do PIB em 2008 está atrelado a uma queda persistente da Produtividade Total dos Fatores (PTF) da economia. Vale à pena destacar que as taxas de desemprego, após 4 anos da crise, são hoje menores que antes da crise e que a utilização da capacidade instalada também é maior hoje. Isso fornece pistas de que ocorreu uma queda da produtividade que se manteve no período. A Figura 3 apresenta a trajetória da PTF no Brasil de 2004 a 2011. Há uma tendência de crescimento da PTF, interrompida com a crise financeira de 2008 e que não chega a retomar o crescimento prévio.

Diante disso, apresentaremos na próxima seção um modelo teórico que fornece as explicações para a relação entre choques externos (como sudden-stops e deterioração nos termos de troca) e as variações na PTF e no produto. Tal modelo será estimado para a economia brasileira para verificar possíveis prescrições de política econômica. 
Figure 2: Trajetória da Produção da Indústria de Transformação

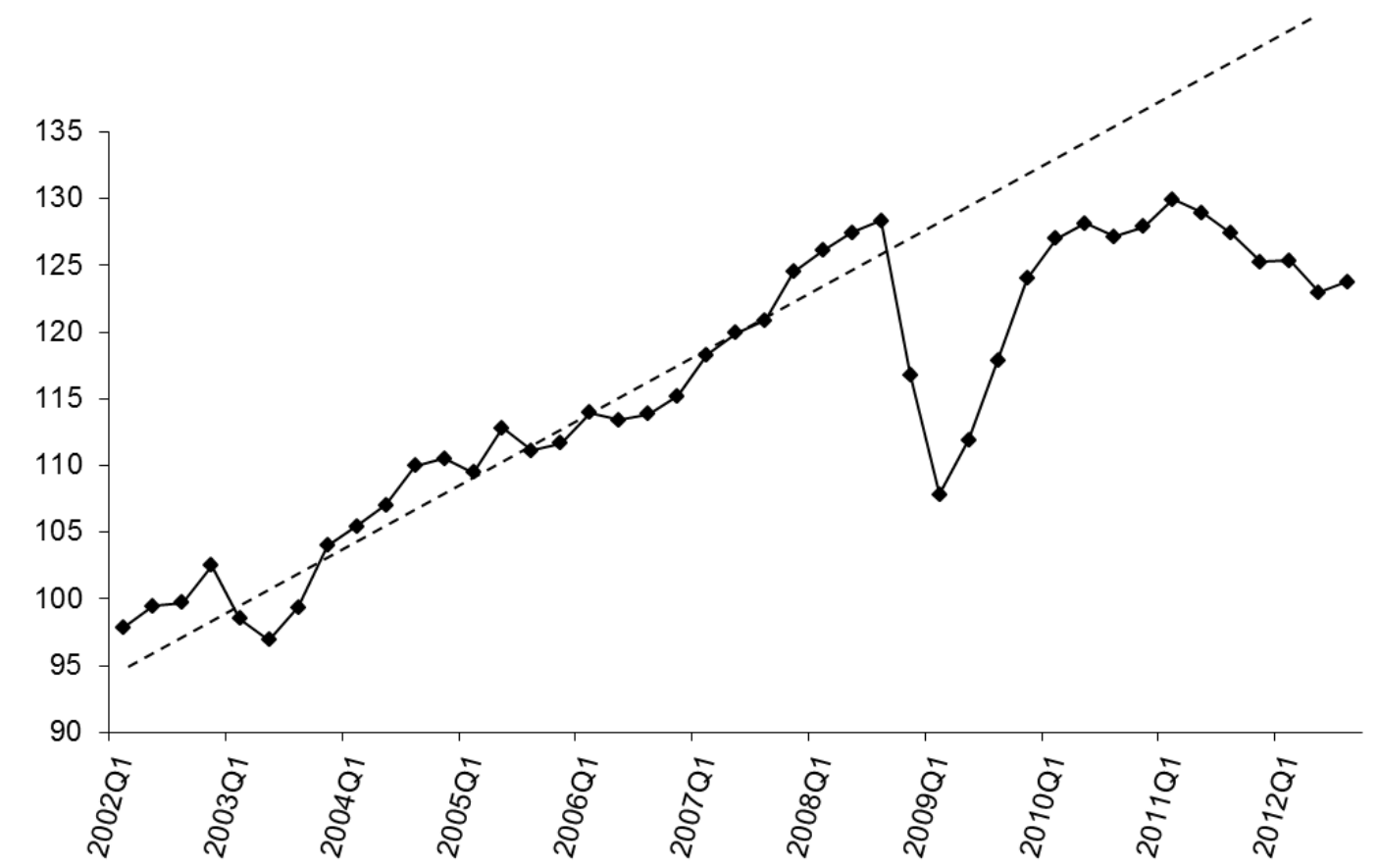

\section{MODELO}

Nesta seção apresentamos o modelo padrão que utilizaremos para avaliar a política monetária em períodos de crise. ${ }^{2} \mathrm{O}$ modelo é uma economia pequena, que importa insumos para a produção e que toma emprestado recursos externos (i.e., recebe um fluxo de capital, que aumenta a liquidez do sistema financeiro). Os termos de troca que as firmas se deparam para importar os insumos e o montante de recursos recebidos seguem um comportamento estocástico. Assim choques nestas variáveis serão as fontes de crises de nossa economia.

O intermediário financeiro recebe recursos do exterior e depósitos das famílias, que repassa às firmas. Ao mesmo tempo a autoridade monetária pode fazer uma injeção de moeda no sistema financeiro por meio de compras ou vendas de títulos públicos em operações de mercado aberto. Como consequência, a taxa de juros cobrada às firmas será diferente da taxa paga às famílias, uma vez que as famílias não podem rever seu portfólio após a intervenção do Banco Central. Assim, o intermediário financeiro tem duas funções:

1) ser o canal de recursos financeiros do exterior e das famílias para as firmas;

2) ser o canal de transmissão da política monetária.

A economia é formada por três tipos de agentes: famílias, firmas e intermediários financeiros. Todos são numerosos de forma a agirem em competição perfeita. Logo, restringimos ao comportamento do agente representativo de cada um deles, e após isso considerar as implicações para equilíbrio geral.

\footnotetext{
${ }^{2}$ Na versão publicada como working paper está incluído um outro modelo mais simples, como forma de deixar clara a intuição da relação entre choques financeiros e a PTF.
} 
Figure 3: Trajetória da PTF

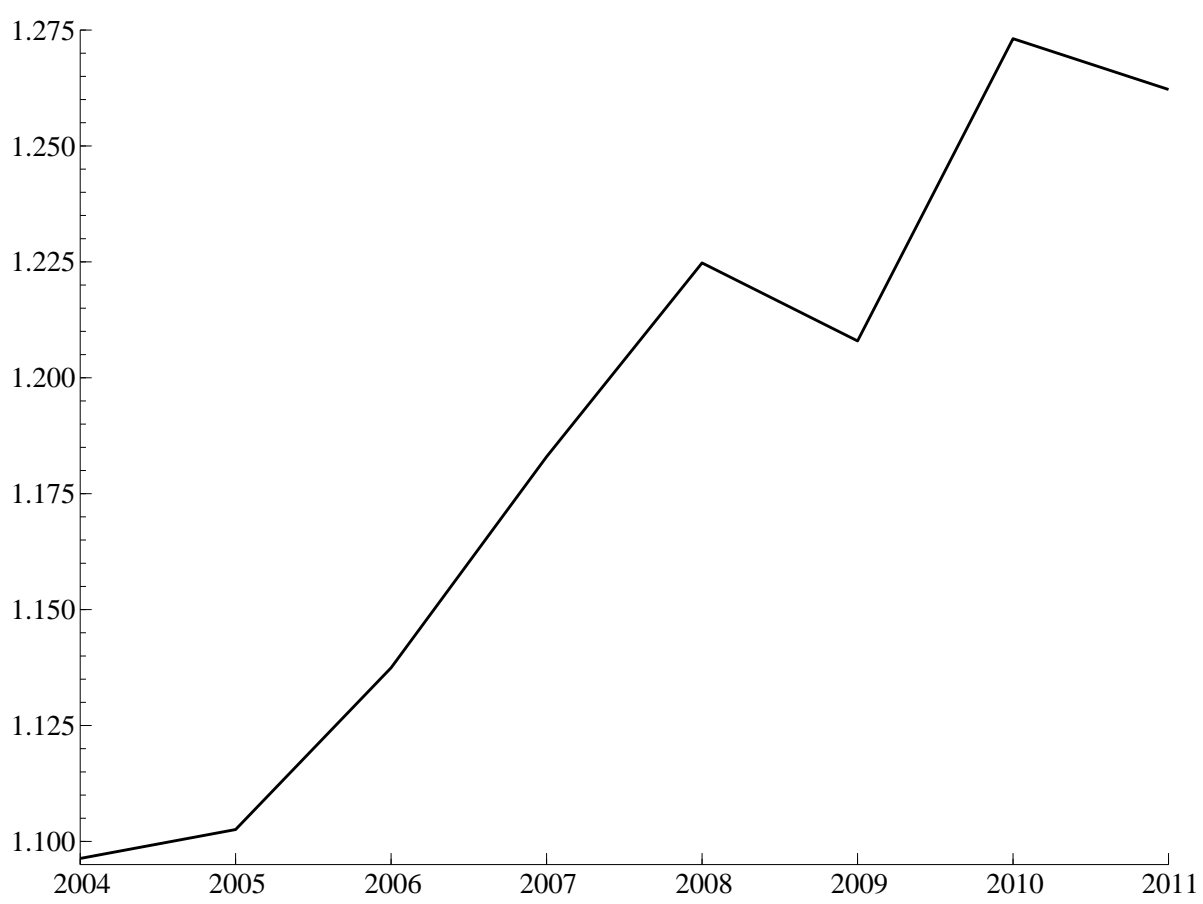

\subsection{Famílias}

As famílias possuem capital e recebem renda do capital, do trabalho e de juros sobre depósitos no sistema financeiro do período anterior. Assim, no início do período as famílias decidem se usarão seus recursos em consumo, investimento em capital, ou em depósitos no sistema financeiro para receber juros no período seguinte.

As famílias vivem infinitamente, e cada família $i$ busca maximizar a função utilidade esperada dada por

$$
E_{t} \sum_{t=0}^{\infty} D^{t}\left\{\ln c_{t}^{i}+Z h_{t}^{i}\right\}
$$

no qual $c_{t}$ é o consumo da família em $t, h$ o trabalho ofertado e $D \in(0,1)$ a taxa de desconto temporal. Na equação (2), utiliza-se a utilidade logarítmica com trabalho indivisível, no qual $Z=\left[b_{0} \frac{\ln \left(1-h_{0}\right)}{h_{0}}\right], b_{0}$ uma constante e $h_{0}$ unidades de trabalho que cada família oferece de trabalho à firma, caso trabalhe.

As famílias estão sujeitas a uma restrição cash-in-advance

$$
P_{t} c_{t}^{i} \leq m_{t-1}^{i}-N_{t}^{i}
$$

e à restrição orçamentária

$$
k_{t+1}^{i}+\frac{m_{t}^{i}}{P_{t}}=w_{t} h_{t}^{i}+r_{t} k_{t}^{i}+(1-\delta) k_{t}^{i}+\frac{r_{t}^{n} N_{t}^{i}}{P_{t}}
$$


no qual $P_{t}$ é o índice de preços em $t$ e $m_{t}$ a moeda que as famílias podem utilizar para consumo ou para depósitos $\left(N_{t}\right)$ no intermediário financeiro, no qual receberão juros $r^{n}$.

Assim, as famílias iniciam o período com o salário recebido $(w)$, o retorno do capital $(r)$, o estoque de capital que não depreciou, e o saldo resultante dos depósitos bancários, e deve decidir entre investir e manter moeda, que será utilizada para novos depósitos ou para consumir durante o período.

\subsection{Firmas}

A firma representativa contrata trabalhadores $(H)$, importa bens intermediários $\left(X_{f}\right)$ e aluga capital $(K)$ sob competição perfeita, maximizando o lucro, e depara-se com uma função de produção dada por

$$
Y_{t}=\lambda_{t} K_{t}^{\theta} X_{f t}^{\alpha} H_{t}^{1-\theta-\alpha}
$$

e maximiza lucros sujeito à restrição orçamentária

$$
Y_{t}=r_{t}^{f} w_{t} H_{t}+r_{t} K_{t}+p_{f t} X_{f t}
$$

Assim, a firma tem de pagar salários, mas também tem de pagar os juros $r^{f}$ pelo capital de giro que tiveram de tomar emprestado para pagarem os salários. Esta mudança significa que choques na taxa de juros alteram as decisões de produção e têm um efeito direto no produto. Além disso, ao importarem bens, as firmas pagam um preço $p_{f}$, que é o inverso dos termos de troca.

A condição de maximização do lucro faz com que as firmas tenham uma demanda por bens intermediários importados $X_{f t}=\alpha \frac{Y_{t}}{p_{f t}}$. Desse modo, podemos reescrever a função de produção como (6)

$$
Y_{t}=\left(\frac{\alpha}{p_{f t}}\right)^{\frac{\alpha}{1-\alpha}} \lambda_{t}^{\frac{1}{1-\alpha}} K_{t}^{\frac{\theta}{1-\alpha}} H_{t}^{\frac{1-\theta-\alpha}{1-\alpha}}
$$

no qual $\beta=1-\theta-\alpha$ e $A_{t}=\left(\frac{\alpha}{p_{f t}}\right)^{\frac{\alpha}{1-\alpha}} \lambda_{t}^{\frac{1}{1-\alpha}}$. Dessa forma, um aumento nos termos de troca (queda em $p_{f}$ ) implica em um aumento da produtividade total dos fatores da economia, $A$.

\subsection{Intermediário financeiro}

O intermediário financeiro opera em um mercado competitivo, recebendo os depósitos das famílias, $N_{t}$, os empréstimos do exterior, $B_{t}$, e as injeções de moeda da autoridade monetária, $\left(g_{t}-1\right) M_{t-1}$, e emprestando esses recursos às firmas.

Na literatura, a restrição de tomada de crédito externo pode ocorrer por causa de restrições de colateral, o que implicaria que a restrição seria endógena de acordo com as condições macroeconômicas internas, como em Arellano e Mendoza (2003), ou por causa de parâmetros externos (como variações nas preferências, tecnologia, produto ou friç̧ões financeiras dos países desenvolvidos), como no modelo de equilíbrio global de Caballero e Panageas (2007), sendo determinado exogenamente. Nós assumimos que o fluxo de capitais é limitado por condições externas uma vez que desejamos interpretar a crise de 2008, por exemplo, que claramente foi causada por um choque externo de liquidez. Como consequência, há uma friç̧ão no sistema financeiro que permite que os bancos tomem emprestado do setor externo e das famílias pagando taxas de juros diferentes, pois a restrição sobre o crédito externo limita tal fonte, fazendo com que os bancos tenham de buscar o crédito das famílias a partir de certo ponto apesar de terem de pagar um juro maior.

Assim, assumindo que os bancos agem sob competição perfeita, eles se deparam com a seguinte restrição orçamentária (condição de lucro zero) 


$$
r_{t}^{f}\left(N_{t}+B_{t}+\left(g_{t}-1\right) M_{t-1}\right)=\int_{0}^{1} r_{t}^{n} N_{t} d i+r^{*} B_{t}=r_{t}^{n} N_{t}+r^{*} B_{t}
$$

ou seja, um aumento da oferta de moeda implica em um efeito de liquidez, diminuindo os juros pagos pelo capital de giro para as firmas.

Assim o valor recebido dos empréstimos das famílias, do setor externo e da autoridade monetária se traduz em pagamento de salários para as firmas de modo que

$$
\left(N_{t}+B_{t}+\left(g_{t}-1\right) M_{t-1}\right)=P_{t} w_{t} H_{t}
$$

tal equação é uma condição de equilíbrio para o mercado financeiro.

Dessa forma uma queda em $B_{t}$ (um sudden-stop) teria um impacto no lado real da economia por reduzir os recursos disponíveis para a contratação de trabalhadores. No modelo, assumiremos que a taxa de juros paga pelos empréstimos externos é uma constante $r^{*}$, enquanto que o fluxo de capitais é uma variável sujeita a choques estocásticos.

\subsection{Política monetária}

O Banco Central (BC) escolhe a taxa de expansão monetária com a finalidade de ajustar o valor da taxa de juros de empréstimos às firmas. A partir das condições (7) e (8), o BC pode escolher a taxa de juros $r_{t}^{f}$ e determinar a taxa bruta de expansão monetária $g_{t}$ pela expressão

$$
g_{t}=\left(\frac{r_{t}^{n}-r_{t}^{f}}{r_{t}^{f}}\right) \frac{N_{t}}{M_{t-1}}\left(\frac{r^{*}-r_{t}^{f}}{r_{t}^{f}}\right) \frac{B_{t}}{M_{t-1}}+1
$$

O crescimento do estoque de moeda é dado por $M_{t}=g_{t} M_{t-1}$.

Por sua vez, o BC determina a taxa de juros desejada dos empréstimos às firmas $r_{t}^{f}$ através de uma regra de Taylor, apresentada pela equação (10), que reage aos desvios da economia ao steady-state.

$$
r_{t}^{f}=a\left(Y_{t}-\bar{Y}\right)+b\left(\pi_{t}-\bar{\pi}\right)+\bar{r}^{f}+t_{t}
$$

no qual as variáveis com traço são os valores de steady-state, $a$ e $b$ os parâmetros de reação da regra de Taylor, $\pi_{t}$ é a inflação da economia e $t_{t}$ o choque de política monetária. Diferentemente do usual, a taxa de juros a ser determinada pela regra de Taylor é uma taxa de juros real e não nominal porque os empréstimos são tomados no início do período e pagos no fim do período. Essa diferença faz com que o parâmetro $b=\hat{b}-1$, no qual $\hat{b}$ seria o parâmetro caso a regra de Taylor regulasse a taxa de juros nominal.

\subsection{Equilíbrio}

O modelo consiste na dinâmica das variáveis $w_{t}, r_{t}, C_{t}, P_{t}, M_{t}, N_{t}, K_{t}, Y_{t}, H_{t}, r_{t}^{n}, r_{t}^{f}$ e $g_{t}$. Considerando as condições de agregação, as condições de primeira ordem das famílias são:

$$
\begin{aligned}
\frac{Z}{w_{t}} & =-D E_{t} \frac{P_{t}}{P_{t+1} C_{t+1}} \\
\frac{1}{w_{t}} & =D E_{t} \frac{r_{t+1}+1-\delta}{w_{t+1}} \\
r_{t}^{n} & =-\frac{w_{t}}{Z C_{t}}=\frac{1}{E_{t} \frac{\beta P_{t} C_{t}}{P_{t+1} C_{t+1}}}
\end{aligned}
$$


As restrições agregadas para as famílias são dadas por

$$
\begin{aligned}
P_{t} C_{t} & =M_{t-1}-N_{t} \\
K_{t+1} & +\frac{M_{t}}{P_{t}}=w_{t} H_{t}+r_{t} K_{t}+(1-\delta) K_{t}+\frac{r_{t}^{n} N_{t}}{P_{t}}
\end{aligned}
$$

Para as firmas, o produto marginal do trabalho iguala-se ao salário real acrescido dos custos de financiamento

$$
r_{t}^{f} w_{t}=\frac{1-\theta-\alpha}{1-\alpha} \frac{Y_{t}}{H_{t}}
$$

e o produto marginal do capital iguala-se à taxa de juros

$$
r_{t}=\frac{\theta}{1-\alpha} \frac{Y_{t}}{K_{t}}
$$

A função de produção é dada por (6) e as condições de equilíbrio para o intermediário financeiro são (7) e (8).

O modelo é log-linearizado em torno do steady-state, originando as equações de espaço de estado que serão estimadas fornecendo os valores dos parâmetros do modelo. A descrição completa do modelo log-linearizado encontra-se no Apêndice A.

\section{ESTIMAÇÃO}

Todas as séries utilizadas foram dessazonalizadas e filtradas pela técnica de Hodrick-Prescott (com o parâmetro de suavização para dados trimestrais no valor de 1600). O gap do produto foi obtido a partir da série de PIB (Produto Interno Bruto) oriunda do IBGE (Instituto Brasileiro de Geografia e Estatística). A taxa de juros de empréstimo às firmas foi obtida a partir da taxa de juros de capital de giro, fornecido pelo $\mathrm{BCB}$. $\mathrm{O}$ indicador de liquidez externa advém do montante líquido da conta financeira ao subtrair o investimento direto estrangeiro líquido, fornecido pelo $B C B$. A partir de tal montante, um índice de liquidez externa foi construído. A série de termos de troca foi obtida a partir da Fundação Centro de Estudos de Comércio Exterior (FUNCEX).

\subsection{Parâmetros calibrados e distribuição das priors}

\subsubsection{Parâmetros calibrados}

Table 1: Parâmetros calibrados

\begin{tabular}{c|c}
\hline Parâmetro & Valor \\
\hline$\delta$ & 0,012 \\
$Z$ & $-2,300$ \\
$D$ & 0,990 \\
$\bar{A}$ & 1,000 \\
$\left(\frac{\bar{B}}{P}\right)$ & 0,065 \\
$\bar{g}$ & 1,016 \\
\hline
\end{tabular}


A calibração do modelo estipula que os valores de $\delta, Z$ e $D$ seguem os trabalhos aplicados ao Brasil de Bonelli e Fonseca (1998), Ferreira e Araújo (1999), Kanczuk (2001, 2002), Ellery et alii (2002), Teles e Andrade (2006) e Souza Sobrinho (2011). O parâmetro de crescimento bruto da moeda transferida para o sistema financeiro no steady-state $\bar{g}$ foi estabelecido como a taxa de inflação trimestral 6,5\% a.a. trimestralizada entre 2000 e 2012. A taxa de juros internacional $r^{*}$ foi calibrada com base na taxa de juros média dos EUA no período. O fluxo de recursos externos em termos reais $\left(\frac{\bar{B}}{P}\right)$ foi estabelecido de modo que se aproximasse de $2,5 \%$ do produto de steady-state da economia, uma média observada para o Brasil. Para normalizar, $\bar{A}=1$. A Tabela 1 apresenta os valores calibrados para os parâmetros.

\subsubsection{Priors}

Os valores médios das priors seguem cálculos para a economia brasileira e valores padrões sugeridos pela literatura. O parâmetro de participação dos insumos importados $\alpha$ foi utilizado como 0,07 que é a média da razão das importações de bens de capital e de bens intermediários em relação ao PIB para o Brasil entre 2000 e 2012. Tal número não se distancia do valor de 0,10 para $\alpha$ obtido por Mendoza (2010). Os parâmetros $\rho_{\lambda}$ e $\sigma_{\lambda}$ foram obtidos de acordo com os trabalhos citados anteriormente, assim como a participação do capital $\frac{\theta}{1-\alpha}$, o que permitiu obter o parâmetro $\theta .^{3}$ Os parâmetros para a regra de Taylor de reação ao produto $(a)$ e à inflação $(b)$ foram assumidos, respectivamente, como 0,5 e 1,5, uma vez que tem sido um resultado padrão em estimações realizadas que a reação do Banco Central que a reação à inflação é muito maior que a reação ao hiato do produto no Brasil.

Por sua vez, os parâmetros para o processo estocástico de $r^{f}, \psi$ e $\eta$ são obtidos através de uma regressão de mínimos quadrados ordinários sobre o gap dessas variáveis. Baseado na série do capital de giro, obtivemos $\rho_{t}=0,75$ e desvio-padrão do resíduo da regressão forneceu $\sigma_{t}=0,01$. A partir da série do inverso dos termos de troca obteve-se o coeficiente do inverso dos termos de troca defasado $\rho_{p}=0,83$ e o resíduo da regressão estabeleceu que $\sigma_{p}=0,01$. Por fim, o índice de liquidez externa levou às estimativas do coeficiente do choque defasado de liquidez externa $\rho_{e}=0,88$ e $\sigma_{e}=1,50$.

As séries utilizadas para a estimação bayesiana são os gaps da liquidez externa $(\tilde{\eta})$, do produto $(\tilde{y})$, do inverso dos termos de troca $(\tilde{\psi})$ e dos juros de capital de giro $\left(\tilde{r}^{f}\right)$.

\subsection{Posteriors estimados}

A solução do modelo é representada em espaço de estado, que é utilizado para computar a função verossimilhança. Usamos uma abordagem bayesiana e escolhemos as distribuições das priors para os parâmetros da função de verossimilhança. A estimação das distribuições das posteriors dos parâmetros é realizada utilizando-se o algoritmo de Metropolis-Hastings.

Utilizamos 4 choques e por isso utilizamos 4 variáveis observadas para fazer a estimação. A amostra vai do primeiro trimestre de 2000 ao último trimestre de 2012. Os resultados das posteriors estimados são apresentados na Tabela 2. As distribuições estimadas das posteriors são apresentadas no Apêndice B.

\section{PROPRIEDADES DO MODELO}

Nesta seção, apresentamos a dinâmica do modelo linearizado usando respostas a impulso, com foco nos choques externos. Na Figura 4, nós observamos as respostas a impulso estimadas para um choque no fluxo de capitais. Neste caso, há uma expansão na entrada de fluxo de capitais no país que causa uma expansão da liquidez da economia que se traduz na redução dos juros para capital de giro $\left(r^{f}\right)$. Tal expansão de liquidez externa implica em um aumento na contratação de trabalhadores $(h)$, uma vez que tal recurso fica barateado, e em um aumento da PTF, uma vez que alivia a fricção financeira, tendo

\footnotetext{
${ }^{3}$ Consequentemente, a participação do trabalho $\frac{\beta}{1-\alpha}=0,60$ de modo a obter o parâmetro $\beta$.
} 
Table 2: Distribuições e parâmetros a priori e a posteriori

\begin{tabular}{l|cc|cc}
\hline \multirow{2}{*}{ Parâmetros } & \multicolumn{2}{|c}{ A priori } & \multicolumn{2}{c}{ A posteriori } \\
\cline { 2 - 5 } & Distribuição & Média & Média & Intervalo \\
\hline$\rho_{t}$ & Beta & 0,75 & 0,77 & {$[0,728 ; 0,818]$} \\
$\rho_{\lambda}$ & Beta & 0,70 & 0,72 & {$[0,679 ; 0,762]$} \\
$\rho_{e}$ & Beta & 0,88 & 0,92 & {$[0,890 ; 0,956]$} \\
$\rho_{p}$ & Beta & 0,83 & 0,80 & {$[0,754 ; 0,849]$} \\
$\beta$ & Normal & 0,54 & 0,62 & {$[0,579 ; 0,666]$} \\
$\theta$ & Beta & 0,36 & 0,32 & {$[0,268 ; 0,375]$} \\
$\alpha$ & Beta & 0,06 & 0,11 & {$[0,074 ; 0,146]$} \\
$a$ & Gama Inversa & 0,50 & 0,18 & {$[0,133 ; 0,230]$} \\
$b$ & Gama Inversa & 1,50 & 2,09 & {$[1,731 ; 2,452]$} \\
$\sigma_{t}$ & Gama Inversa & 0,01 & 0,010 & {$[0,006 ; 0,013]$} \\
$\sigma_{\lambda}$ & Gama Inversa & 0,01 & 0,004 & {$[0,003 ; 0,005]$} \\
$\sigma_{e}$ & Gama Inversa & 1,50 & 1,923 & {$[1,638 ; 2,205]$} \\
$\sigma_{p}$ & Gama Inversa & 0,01 & 0,017 & {$[0,014 ; 0,019]$} \\
\hline \multicolumn{2}{|c}{}
\end{tabular}

como resultado um aumento do retorno marginal do capital $(r)$. Dessa forma, a economia experimenta um aumento do produto $(y)$ e do consumo $(c)$. Um choque negativo de liquidez externa teria um efeito simétrico, ou seja, uma crise de liquidez externa implicaria em um aumento dos juros de capital de giro, seguido de uma queda da produção e da contratação de trabalhadores. Tais movimentos foram verificados por ocasião da crise de 2008 no país.

Na Figura 5, avaliamos as respostas a um impulso negativo nos termos de troca (i.e. um choque positivo em $p_{f}$ ). Tal choque implica em um aumento do custo de produção, uma vez que o bem intermediário importado fica mais caro, e reduz a PTF, ao causar ineficiência alocativa. Como consequência, há uma redução do produto marginal do capital e do trabalho, reduzindo-se $r$ e $h$, e por consequência, a produção $y$.

A Figura 6 apresenta as respostas a um impulso monetário expansionista, diminuindo de imediato os juros de capital de giro, seguido de um aumento em face da regra de reação do Banco Central. A expansão monetária não é neutra uma vez que há friç̧ões financeiras que fazem com que o custo do trabalho dependa da política monetária. Assim, a diminuição dos juros tem uma implicação no aumento de contratação de trabalhadores o que implica em um aumento do produto e do retorno marginal do capital.

\section{APLICAÇÕES}

Uma forma de avaliar a importância dos choques externos sobre as flutuações do produto na economia é verificar a decomposição dos choques no produto, disposta na Figura 7.

Nós observamos na decomposição dos choques, que as variações de fluxo de capitais não representam um componente relevante para explicar as flutuações do produto na maior parte do período analisado, mas que a crise de 2008 pode ser explicada de forma preponderante pela queda de liquidez externa, deixando claro que choques abruptos nessa variável têm um impacto relevante na economia. 
Figure 4: Respostas a Impulso no Fluxo de Capitais
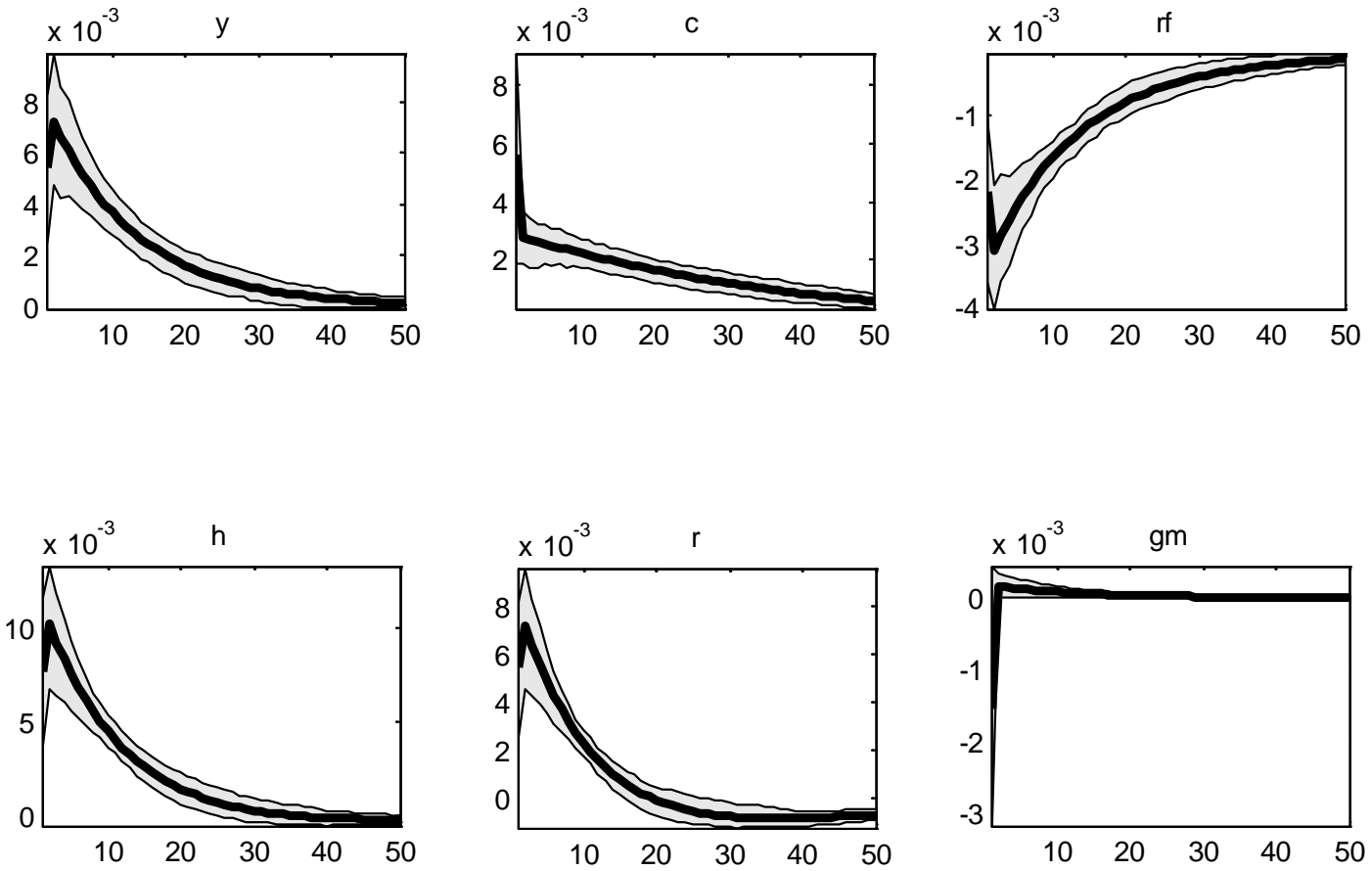

Figure 5: Respostas a Impulso no Inverso dos Termos de Troca
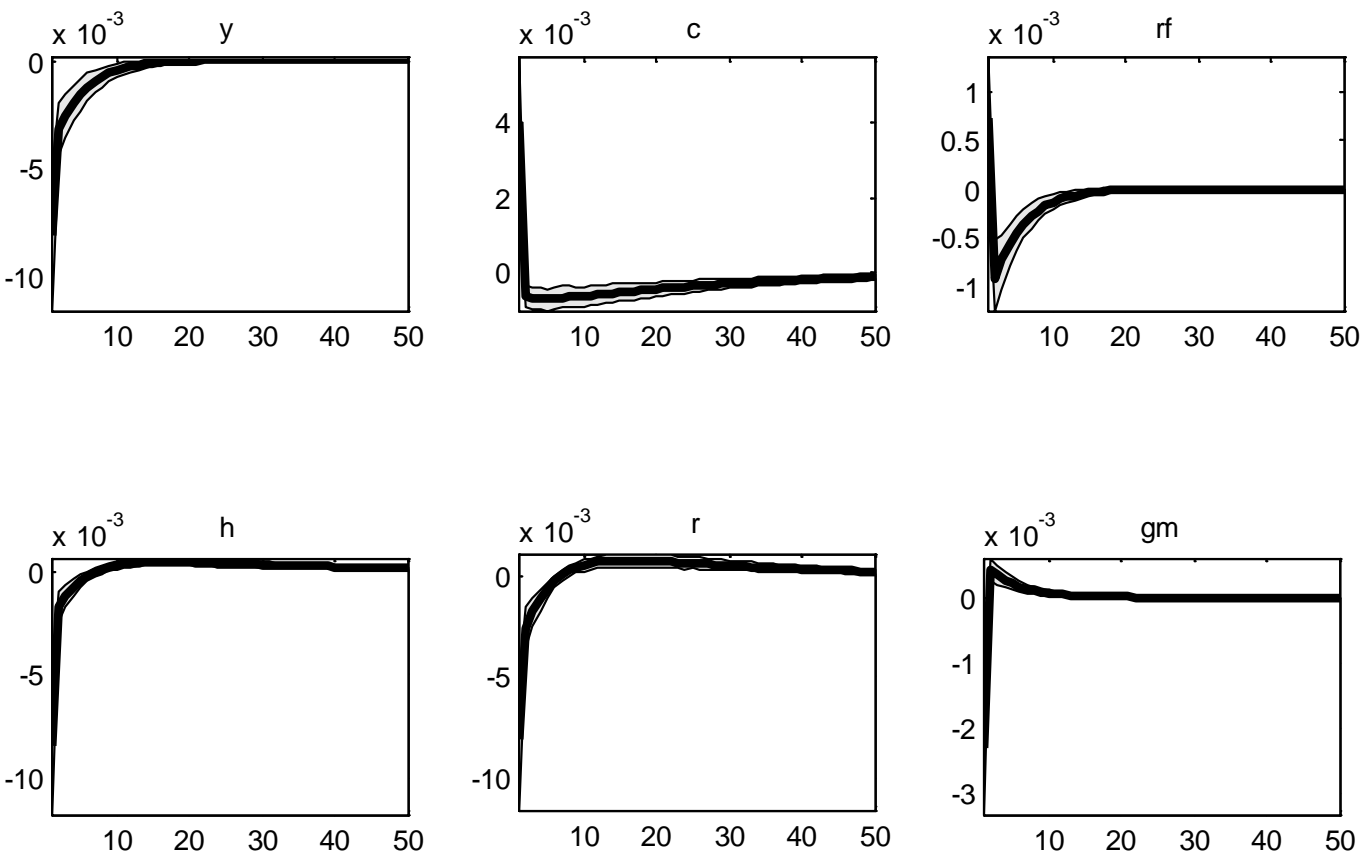
Figure 6: Respostas a Impulso Monetário Expansionista
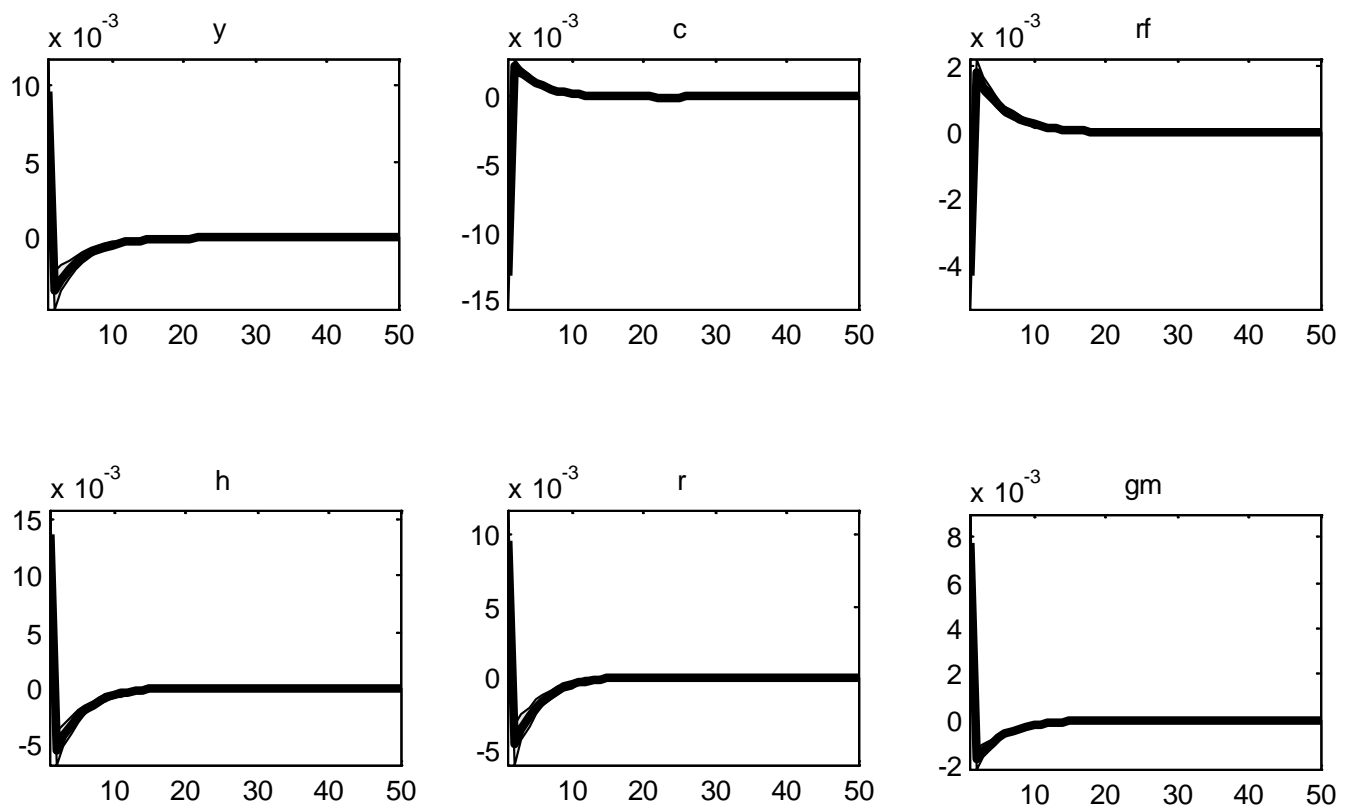

Figure 7: Decomposição dos Choques de Produto

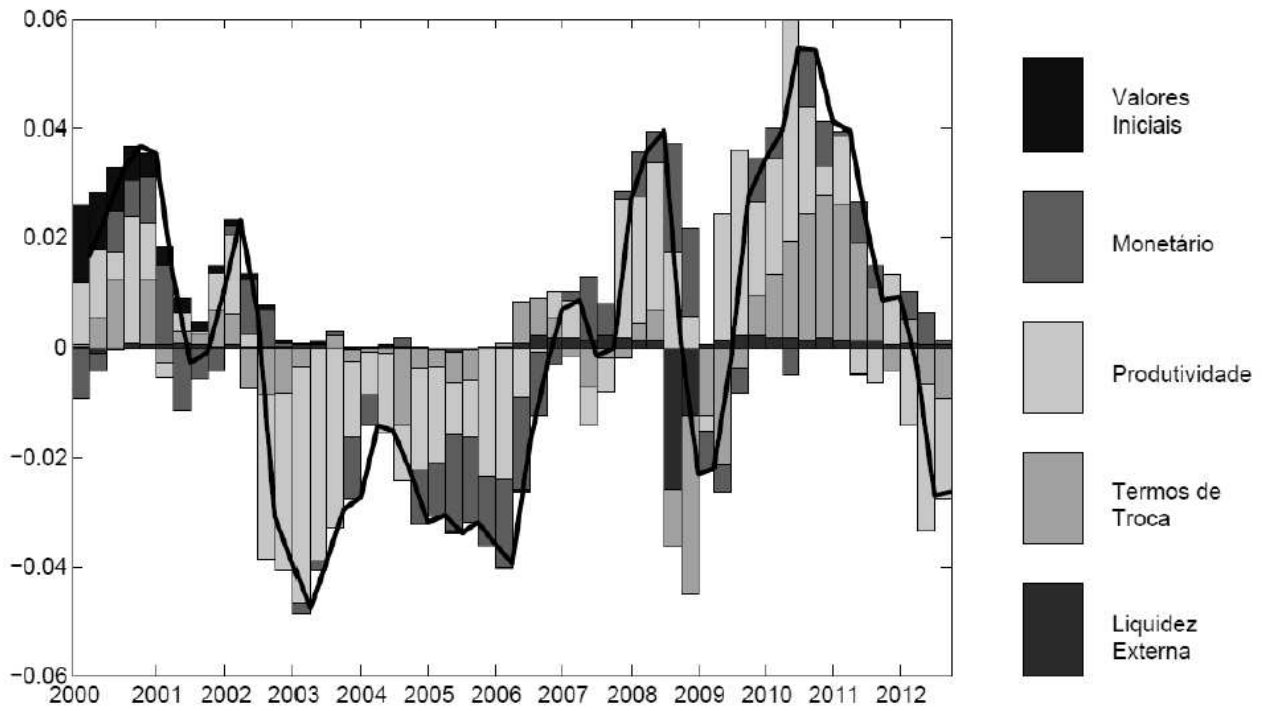


Por outro lado, as variações nos termos de troca representam uma parcela significativa da explicação das variações do produto, sobretudo no período pós-crise, de forma que nossos resultados corroboram com outros apresentados na literatura, como em Cardoso e Teles (2010). Nesse sentido, fica claro aqui que a correlação entre termos de troca e flutuações do produto podem ser explicadas por um canal de variações na produtividade da economia.

Já os choques inesperados de política monetária se destacam no período entre 2005 e 2007 quando houve uma política monetária mais conservadora, refletindo-se de forma significativa em uma redução do produto. Ao mesmo tempo, os resultados para o período pós-crise refletem a falta de capacidade da política monetária expansionista adotada nesse período de ter um impacto positivo no produto de forma relevante.

Figure 8: Hiato da PTF Previsto vs Observado

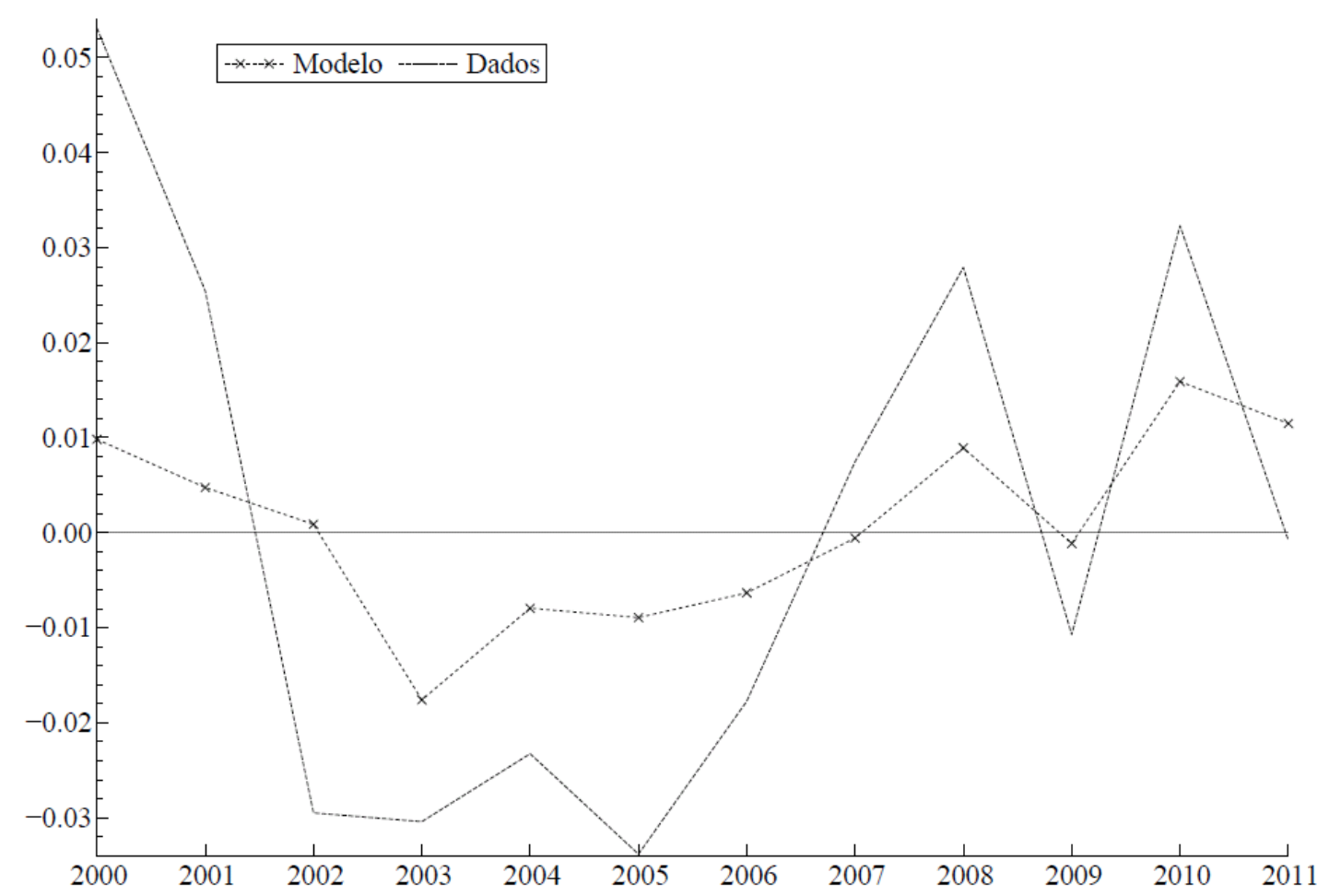

Uma forma de avaliarmos a capacidade do modelo de explicar os movimentos da economia brasileira, e especialmente as variações de curto prazo da PTF, é compararmos os valores previstos para a série da PTF pelo modelo com os valores calculados a partir do resíduo de Solow sem tendência. Tal comparação é realizada a partir na Figura 8. A figura mostra que a série prevista segue a mesma direção da observada, embora com menos flutuações. A correlação das duas séries é de 0,79. Assim, podemos dizer que o modelo é capaz de produzir um ajuste bastante próximo do observado.

\section{IMPLICAÇÕES PARA POLÍTICA MONETÁRIA}

Para analisar as implicações de política monetária, realizamos um exercício de verificar como diferentes regras de política monetária impactam o bem estar com base nos parâmetros estruturais estimados. Os resultados deste exercício encontram-se na Tabela 3. A coluna da regra 1 apresenta os 
resultados obtidos ao se utilizar à regra de reação estimada para o período, onde a reação a inflação mostrou-se bem maior que a de variações no hiato do produto. As linhas mostram como a economia reagiria sob circunstâncias diferentes, a saber, diante de todos os choques incluídos no modelo, ou diante de cada choque específico.

Table 3: Avaliação de diferentes regras de Taylor de acordo com o bem-estar

\begin{tabular}{l|c|c|c|c|c|c}
\hline \multirow{2}{*}{} & \multicolumn{7}{|c}{ Regras de Taylor } \\
\cline { 2 - 7 } Pesos & & 1 & 2 & 3 & 4 & 5 \\
& $Y$ & 0,18 & 0,00 & 0,40 & 0,18 & 0,18 \\
& $\pi$ & 2,09 & 2,09 & 2,09 & 1,70 & 2,50 \\
\hline Choques & & & & & & \\
\hline Todos & & 76,421 & 76,474 & 76,400 & 76,428 & 76,422 \\
Liquidez Externa & & 77,799 & 77,902 & 77,729 & 77,755 & 77,843 \\
Termos de Troca & & 75,733 & 75,698 & 75,766 & 75,734 & 75,732 \\
Produtividade & & 76,099 & 76,101 & 76,098 & 76,099 & 76,099 \\
Monetário & & 76,068 & 76,068 & 76,069 & 76,067 & 76,069 \\
\hline
\end{tabular}

As colunas das regras de 2 a 5 mostram qual seria o resultado de alterar os parâmetros da regra de Taylor, aumentando ou diminuindo o rigor com relação à inflação e/ou ao hiato do produto. Tais resultados ajudam a interpretar o modelo, pois fornecem como as distorções incorporadas são magnificadas por diferentes choques e como as reações do Banco Central podem ser úteis para controlá-las.

Nesse respeito, as regras de Taylor são preferíveis para aumentar o bem-estar em relação a regras de crescimento constante do agregado monetário, pois estabilizam o juro nominal, que é a fonte de distorção do bem estar em última instância (ver Carlstrom e Fuerst, 1995). No nosso caso, os choques de liquidez externa têm um impacto distorcivo no montante de liquidez do sistema financeiro, e assim sendo uma espécie de choque nominal com efeitos reais. Diante disso, as regras de reação com maior peso à inflação são as que têm melhor desempenho para aumentar o bem estar. Por outro lado, quando a origem do choque é nos termos de troca, a regra que implica em um valor mais alto do bem estar é a que reage mais fortemente a variações no hiato do produto.

\section{CONCLUSÕES}

Neste trabalho, verificamos como crises causadas por choques externos, como sudden-stops e depreciação dos termos de trocas, afetariam a economia brasileira no período 2000-2012. Para tanto o presente artigo propõe um modelo simples, de cash-in-advance e working capital, no qual choques externos são capazes de afetar a decisão de alocação de fatores de produção, e assim a produtividade total dos fatores da economia.

O modelo é estimado, de forma que a importância dos choques externos pode ser avaliada para o período. O ajuste do modelo é ilustrado por produzir uma série de variações na PTF similar à observada, apesar de não incluir na estimação como variável observada a variável de produtividade, horas trabalhadas, ou de estoque de capital.

Ao avaliar as implicações do modelo, foi possível notar que embora variações na liquidez externa não tenham um impacto relevante no produto na maior parte do período analisado, foi o choque mais 
relevante para explicar a crise de 2008. Ao mesmo tempo, os choques nos termos de troca têm uma importância na recuperação do produto, principalmente no período pós-2008.

\section{BIBLIOGRAPHY}

Acemoglu, D. \& Ventura, J. (2002). The world income distribution. The Quarterly Journal of Economics, 117:659-694.

Arellano, C. \& Mendoza, E. (2003). Credit frictions and 'sudden stops' in small open economies: An equilibrium business cycle framework for emerging markets crises. In Altug, S., Chadha, J., \& Nolan, C., editors, Dynamic Macroeconomic Analysis: Theory and Policy in General Equilibrium. Cambridge University Press.

Bonelli, R. \& Fonseca, R. (1998). Ganhos de produtividade e de eficiência: Novos resultados para a economia brasileira. Pesquisa e Planejamento Econômico, 28:273-314.

Caballero, R. \& Panageas, S. (2007). A global equilibrium model of sudden stops and external liquidity management. Manuscript, MIT, Department of Economics.

Calvo, G. A., Izquierdo, A., \& Talvi, E. (2006). Sudden stops and phoenix miracles in emerging markets. American Economic Review, 96:405-410.

Cardoso, E. \& Teles, V. K. (2010). A brief history of Brazil's growth. In Mello, L., editor, Growth and Sustainability in Brazil, China, India, Indonesia and South Africa, pages 19-50. OECD.

Carlstrom, C. T. \& Fuerst, T. S. (1995). Interest rate rules vs. money growth rules a welfare comparison in a cash-in-advance economy. Journal of Monetary Economics, 36:247-267.

Cerra, V. Saxena, S. C. (2008). Growth dynamics: The myth of economic recovery. American Economic Review, 98:439-57.

Cooley, T. \& Hansen, G. D. (1989). The inflation tax in a real business cycle model. American Economic Review, 79:733-48.

Ellery, R. G., gomes, V., \& Sachsida, A. (2002). Business cycle fluctuations in Brazil. Revista Brasileira de Economia, 56:269-308.

Ferreira, P. C. \& Araújo, C. H. V. (1999). Reforma tributária, efeitos alocativos e impactos de bem-estar. Revista Brasileira de Economia, 53:133-166.

Kanczuk, F. (2001). Business cycle in a small open Brazilian economy. Economia Aplicada, 5:455-470.

Kanczuk, F. (2002). Juros reais e ciclos reais brasileiros. Revista Brasileira de Economia, 56:249-267.

Mendoza, E. G. (1995). The terms of trade, the real exchange rate, and economic fluctuations. International Economic Review, 36:101-37.

Mendoza, E. G. (2010). Sudden stops, financial crises, and leverage. American Economic Review, 100:1941-66.

Neumeyer, P. A. \& Perri, F. (2005). Business cycles in emerging economies: The role of interest rates. Journal of Monetary Economics, 52:345-380.

Pratap, S. \& Urrutia, C. (2012). Financial frictions and total factor productivity: Accounting for the real effects of financial crises. Review of Economic Dynamics, 15:336-358. 
Souza Sobrinho, N. F. (2011). The role of interest rates in the Brazilian business cycles. Revista Brasileira de Economia, 65:315-336.

Teles, V. K. \& Andrade, J. (2006). Reformas tributária e previdenciária e a economia brasileira no longo prazo. Revista Brasileira de Economia, 60:87-107. 


\section{A. APÊNDICE A - MODELO COMPLETO LOG-LINEARIZADO}

A partir das equações do modelo, obtemos o estado estacionário, e em seguida as variáveis log-linearizadas em torno do estado estacionário. As equações para se obter as variáveis no estado estacionário $\left(\frac{\bar{M}}{P}, \bar{N} \frac{\bar{P}}{\bar{P}}, \overline{r^{f}}, \bar{C}, \bar{H}\right)$ são

$$
\begin{gathered}
\overline{r^{f}}=-\left[\left(\frac{\theta}{\bar{r}}\right)^{\frac{\theta}{1-\alpha}} \beta^{\frac{\beta}{1-\alpha}} \bar{A}\right] \frac{D}{\bar{C} \bar{g} Z} \\
\left(\overline{r^{n}}-\overline{r^{f}}\right)(\overline{\bar{N}} \bar{P})+\left(\overline{r^{*}}-\overline{r^{f}}\right)\left(\frac{\bar{B}}{P}\right)=\overline{r^{f}}\left[1-\frac{1}{\bar{g}}\right]\left(\overline{\frac{M}{P}}\right) \\
\left(\overline{\frac{N}{P}}\right)+\left[1-\frac{1}{\bar{g}}\right]\left(\frac{\bar{M}}{P}\right)+\left(\frac{\bar{B}}{P}\right)=-\frac{\bar{C} \bar{g} Z \bar{H}}{D} \\
\bar{C}=\frac{1}{\bar{g}}\left(\overline{\frac{M}{P}}\right)-\left(\overline{\frac{N}{P}}\right) \\
\left(\overline{\frac{M}{P}}\right)=\frac{\bar{g}}{\bar{D}}\left(\frac{\bar{N}}{P}\right)+\left[(\bar{r}-\delta)\left(\frac{\theta}{\bar{r}} \bar{A}\right)^{\frac{1-\alpha}{\beta}}-\frac{\bar{C} \bar{g} Z}{D}\right] \bar{H}
\end{gathered}
$$

O próximo passo para permitir computar a dinâmica é a obtenção das equações log-linearizadas. A partir das equações de equilíbrio do modelo, obtemos as formas log-linearizadas a seguir.

$$
\begin{aligned}
& 0=\tilde{w}_{t}+\tilde{P}_{t}-\tilde{P}_{t+1}-\tilde{C}_{t+1} \\
& 0=\tilde{w}_{t}-\tilde{w}_{t+1}+D \bar{r} \tilde{r}_{t+1} \\
& 0=\tilde{C}_{t}-w_{t}+\tilde{r}_{t+1}^{n} \\
& 0=\bar{C} \tilde{C}_{t}+\bar{C} \tilde{P}_{t}-\frac{1}{\bar{g}}\left(\overline{\frac{M}{P}}\right) \tilde{M}_{t-1}+\left(\overline{\frac{N}{P}}\right) \tilde{N}_{t} \\
& 0=(\overline{\bar{M}} \bar{P}) \tilde{M}_{t}+\bar{K} \tilde{K}_{t+1}+\left[\overline{r^{n}}\left(\overline{\frac{N}{P}}\right)-(\overline{\bar{M}} \bar{P})\right] \tilde{P}_{t}-\bar{w} \bar{H}\left(\tilde{w}_{t}+\tilde{H}_{t}\right) \\
& -\bar{r} \bar{K} \tilde{r}_{t}-\bar{K} \tilde{K}_{t}(\bar{r}+1-\delta)-\overline{r^{n}}\left(\overline{\bar{N}} \frac{\bar{N}}{P} \tilde{N}_{t}-\overline{r^{n}}\left(\overline{\frac{N}{P}}\right) \tilde{r}_{t}^{n}\right. \\
& 0=\tilde{Y}_{t}-\tilde{H}_{t}-\tilde{r}_{t}^{f}-\tilde{w}_{t} \\
& 0=\tilde{r}_{t}-\tilde{Y}_{t}+\tilde{K}_{t} \\
& 0=\tilde{Y}-\tilde{A}_{t}-\frac{\theta}{1-\alpha} \tilde{K}_{t}-\frac{\beta}{1-\alpha} \tilde{H}_{t}
\end{aligned}
$$




$$
\begin{aligned}
& \overline{r^{n}}\left(\overline{\frac{N}{P}}\right) \tilde{N}_{t}+\overline{r^{n}}\left(\overline{\frac{N}{P}}\right) \tilde{r}_{t}^{n}+\overline{r^{*}}\left(\overline{\frac{B}{P}}\right) \tilde{B}_{t}-\left[\overline{r^{N}}\left(\overline{\frac{N}{P}}\right)+\overline{r^{*}}\left(\overline{\frac{B}{P}}\right)\right] \tilde{P}_{t} \\
& =\overline{r^{f}} \bar{w} \bar{h}\left(\tilde{r}_{t}^{f}+\tilde{w}_{t}+\tilde{H}_{t}\right) \\
& (\overline{\bar{N}} \bar{P}) \tilde{N}_{t}+\left(\overline{\frac{M}{P}}\right)\left(1-\frac{1}{\bar{g}}\right) \tilde{M}_{t-1}+\left(\overline{\frac{M}{P}}\right) \tilde{g}_{t}+(\overline{\bar{B}} \bar{P}) \tilde{B}_{t}=\bar{w} \bar{H}\left(\tilde{w}_{t}+\tilde{H}_{t}+\tilde{P}_{t}\right) \\
& 0=\tilde{M}_{t}-\tilde{g}_{t}-\tilde{M}_{t-1} \\
& 0=a \bar{Y} \tilde{Y}_{t}+b \bar{g} \tilde{\pi}_{t}-\overline{r^{f}} \tilde{r}_{t}^{f}+\tilde{t}_{t}
\end{aligned}
$$

no qual as variáveis com $\sim$ representam o desvio da variável em relação ao steady-state, $\tilde{\pi}_{t}=\tilde{P}_{t}-$ $\tilde{P}_{t-1}, \tilde{A}_{t}=-\frac{\alpha}{1-\alpha} \tilde{p}_{f t}+\frac{1}{1-\alpha} \tilde{\lambda}_{t}, \tilde{B}_{t}=\tilde{\eta}_{t}$ e $\tilde{p}_{f t}=\tilde{\psi}_{t}$

Além disso, as equações dos processos estocásticos de $\tilde{\eta}_{t}, \tilde{\psi}_{t}, \tilde{\lambda}_{t}$ e $\tilde{t}_{t}$ são:

$$
\begin{gathered}
\tilde{\lambda}_{t}=\rho_{\lambda} \tilde{\lambda}_{t-1}+\varepsilon_{t}^{\lambda} \\
\tilde{t}_{t}=\rho_{t} \tilde{t}_{t-1}+\varepsilon_{t}^{t} \\
\tilde{\eta}_{t}=\rho_{e} \tilde{\eta}_{t-1}+\varepsilon_{t}^{e} \\
\tilde{\psi}_{t}=\rho_{p} \tilde{\psi}_{t-1}+\varepsilon_{t}^{p}
\end{gathered}
$$




\section{B. APÊNDICE B - POSTERIORS ESTIMADOS}

Figura B.1: Posteriors Estimados

SE

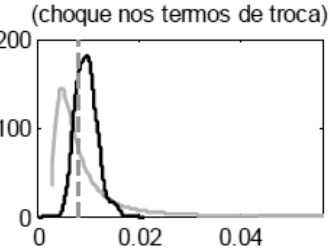

SE

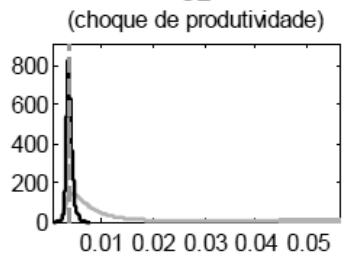

rhoe

(AR(1) do fluxo de capitais)

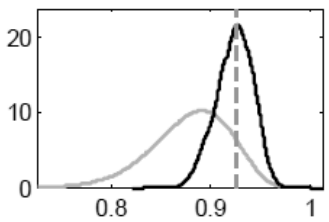

SE

(choque no fluxo de capitais)

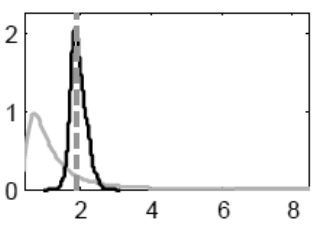

rhot

(AR(1) do choque monetário)

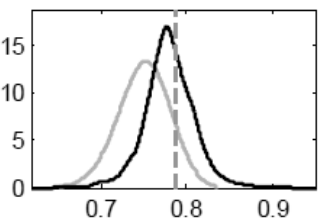

hop

(AR(1) dos termos de troca)

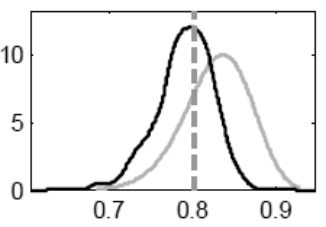

SE

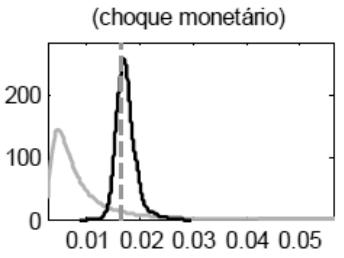

rhol

(AR(1) do choque de produtividade)

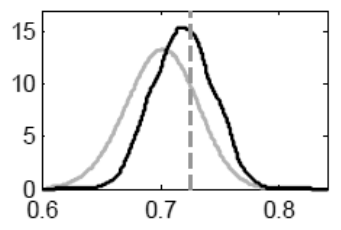

beta

(Labor share)

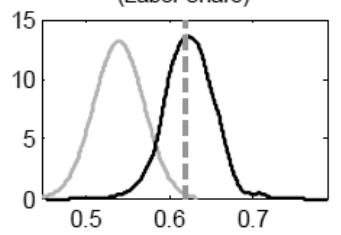


Figura B.1: Posteriors Estimados (continuação)

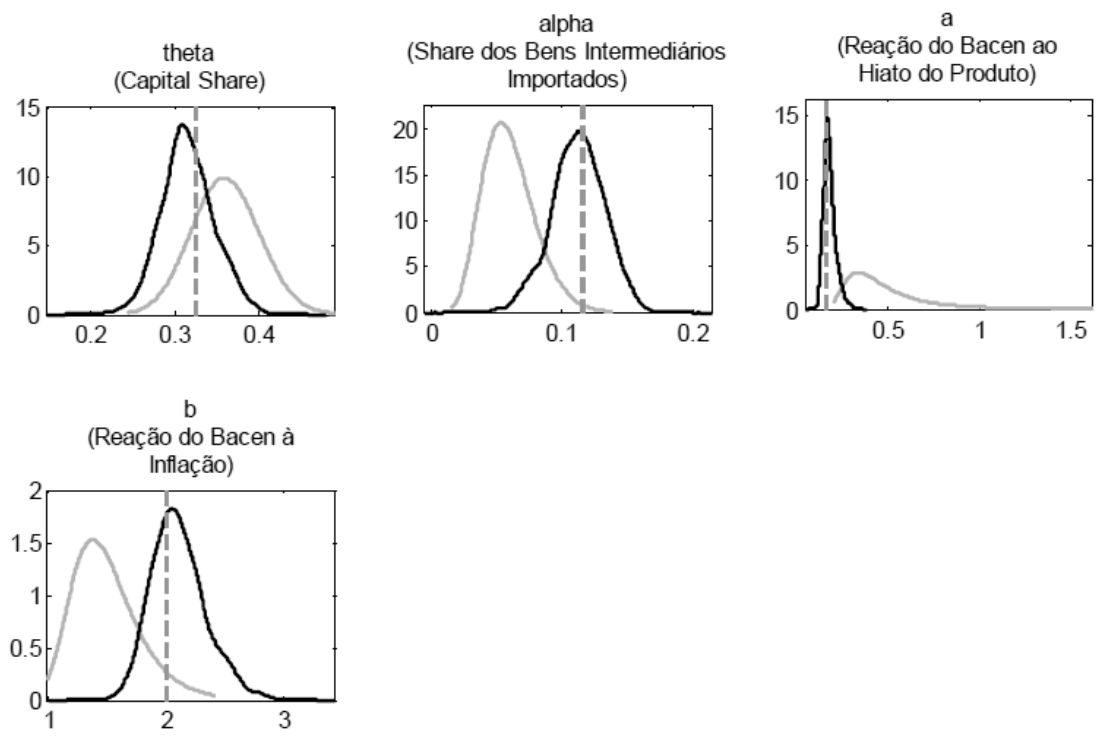

